\title{
A Bayesian Network for Autonomous Sensor Control during Polar Ice Sheet Measurements
}

\author{
Sudha Sivashanmugam \\ Dept. of Electrical Engineering and Computer Science \\ Information and Telecommunication Technology Center \\ The University of Kansas \\ 2335 Irving Hill Road, Lawrence, KS 66045, USA \\ ssivasha@ittc.ku.edu
}

\author{
Costas Tsatsoulis \\ Dept. of Electrical Engineering and Computer Science \\ Information and Telecommunication Technology Center \\ The University of Kansas \\ 1520 West $15^{\text {th }}$ St., Room 2001, Lawrence, KS 66045, USA \\ tsatsoul@ittc.ku.edu
}

\begin{abstract}
The PRISM (Polar Radar for Ice Sheet Measurements) project at the University of Kansas is developing intelligent radar sensors for the measurement and study of the mass balance of the polar ice sheets. An important component of PRISM is an intelligent, autonomous Synthetic Aperture Radar that can reason about its operating mode (monostatic vs. bistatic) and frequency, based on a variety of environmental and sensorrelated factors. The PRISM sensors are placed on autonomous robotic vehicles ("rovers") that use the sensor and environmental information to decide about what paths to traverse, how to traverse them, and at what speeds. In our work, we have implemented the reasoning component of the autonomous radar and the rovers, using intelligent agents and Bayesian networks. This implementation is the first ever of a dynamically modifying adaptive radar and mobile data collection system based on autonomous rovers for accurate polar ice sheet measurements.
\end{abstract}

Keywords- polar ice sheet measurements; autonomous radar and rover control; intelligent agents; Bayesian networks; probabilistic inference;

\section{INTRODUCTION}

The Polar Radar for Ice Sheet Measurements (PRISM) project at the University of Kansas is developing autonomous, mobile, intelligent radar sensors for the measurement and study of the mass balance of the Greenland and Antarctica ice sheets. Based on these measurements, researchers involved in the project will provide data to the scientific community to improve the understanding of ice sheet dynamics and the ability to create ice sheet models that would determine the contribution of melted ice to sea level rise.

The primary sensors developed consist of a Synthetic Aperture Radar (SAR) and a wideband, dual-mode radar. The SAR can operate in bistatic or monostatic mode and at three different frequencies $-60,150$ and $350 \mathrm{MHz}$. It is used to generate two-dimensional reflectivity maps of the bed for determining basal conditions, including the presence and distribution of basal water. The wideband, dual-mode radar employs a radar depth sounder for measuring ice thickness and mapping deep internal layers and an accumulation radar for mapping near-surface internal layers. The radar sensors are integrated into a complete information system consisting of two vehicles - an automated rover and a manned (tracked) vehicle. These vehicles are configured with the necessary communication and navigation systems to collect data from the sensors and route them back to a central location for further processing, analysis, and storage.

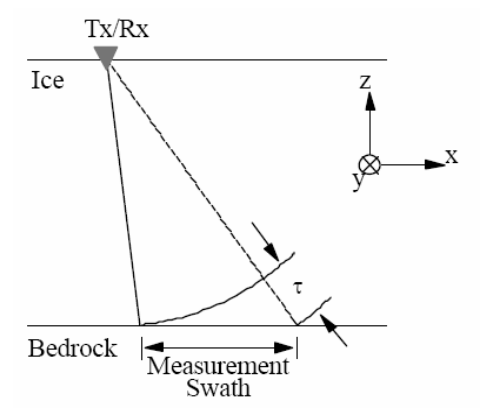

Monostatic SAR mode

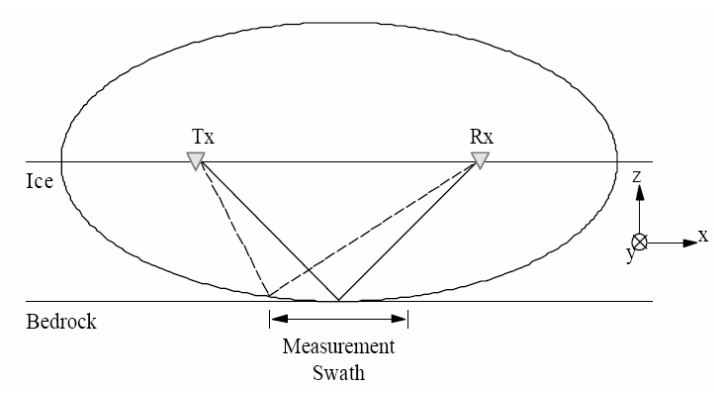

Bistatic SAR mode

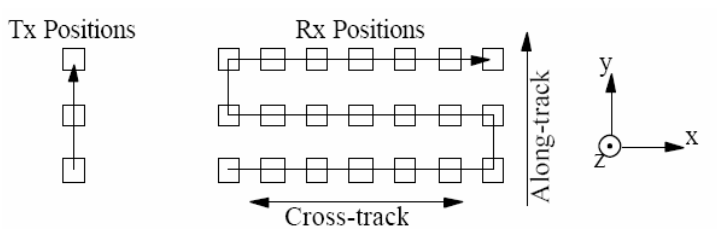

Tx/Rx positions, cross-track/along-track movements of rover in bistatic SAR mode

Figure 1. Monostatic/Bistatic SAR (adapted from [1]). 
The SAR transmitter, the monostatic SAR receiver, and one dual-mode radar are operated on the manned vehicle. The bistatic SAR receiver and another dual-mode radar are operated on the automated rover. The rover moves in nearly straightline paths (along the base vehicle) during monostatic SAR measurements and makes cross-track transects during bistatic SAR measurements. Figure 1 illustrates the monostatic/bistatic SAR measurements, and shows the transmitter/receiver positions and the rover transects in bistatic SAR mode.

The PRISM sensors are equipped with onboard intelligence that provides them with the ability to autonomously decide where and how to measure. The onboard intelligent system must dynamically determine the appropriate operating mode and frequency of the SAR, distance of the vehicles from the center of the measurement swath, cross-track transect length and along-track spacing of the rover in bistatic SAR mode, the speed and scan path (sequence of way points) of the rover, based on the measurements made by one or more of the sensors in real-time. The system must fuse any recently collected sensor data with a priori information, for example from satellites like RADARSAT, and other image data to determine the "optimum" sensor configuration for imaging the bed. It must handle uncertainty in the environment and sensor data, and make decisions depending on multiple criteria like potential scientific benefits, available system power and computing resources, wear and tear, and quality of judgment in terms of confidence in the different data inputs, and so on.

We have developed an intelligent sensor and rover controlling system based upon a multiagent collaborative architecture that involves a number of distinct data collection and data dissemination agents that function continuously and autonomously in a distributed computing framework. The agents that control the rover and the radar sensors consult a probabilistic inference engine to make environmental and sensor-related decisions. We have used Bayesian networks to model dependencies between the different decision inputs and outputs of the intelligent system. Explanation rules (criteria) for fusing multiple data sets and making decisions have been coded in terms of conditional probabilities. Decision-making is based on the axioms of classical probability theory.

\section{BAYESIAN NETWORKS}

A Bayesian network can be considered as an important paradigm of expert systems. It is a probabilistic system that is used to model a domain containing uncertainty in some manner. Based on Jensen's definition, a Bayesian network consists of the following [2]:

- A set of variables (nodes) and a set of directed edges between the variables.

- Each variable has a finite set of mutually exclusive states.

- The variables together with the directed edges form a directed acyclic graph. (A directed graph is acyclic if there is no directed path $\mathrm{A}_{1} \rightarrow \ldots \rightarrow \mathrm{A}_{n}$ such that $A_{1}=A_{n}$ )
- Edges between nodes reflect the dependency (cause-effect) relations within the domain. These effects are normally not completely deterministic. The strength of an effect is modeled as a probability. Hence, to each variable $A$ with parents $B_{1}, \ldots, B_{n}$ in the Bayesian network, there is attached the conditional probability table $P\left(A \mid B_{1}, . ., B_{n}\right)$. If $A$ has no parents, then the table reduces to unconditional probabilities $P(A)$.

Fundamentally, Bayesian networks are used to make probabilistic inferences - the process of computing / updating the posterior marginal probability distributions of a set of variables of interest when information or evidence of other variables in the network comes in. Making an inference in a Bayesian network is based on the notion of evidence propagation. The mathematical basis for this is the Bayes theorem:

$$
P(A \mid B)=P(B \mid A) . P(A) / P(B)
$$

In a Bayesian network, any node can receive information. The inference method does not distinguish between inference in or opposite to the direction of edges. Also, simultaneous input of information into several nodes will not affect the updating algorithm. Unlike the updating methods of rule-based systems, the updating method of Bayesian networks uses a global perspective, and if the model and information are correct, it can be proved that the method calculates the new probabilities correctly according to the theory of probability [3][6].

\section{THE PRISM BAYESIAN NETWORK}

The decision-making process of the PRISM intelligent system is highly complex and dynamic. Apart from any source information collected by the sensors in real time and other a priori information, the decision-making process is influenced by several other criteria. While a small part of the decisionmaking process is deterministic in nature, a major part of it is probabilistic. It is these probabilistic rules of decision-making that have been coded into conditional probabilities, and the dependencies between the decision variables have been represented as a Bayesian network.

In the PRISM Bayesian network, the leaf nodes represent decision outputs, the parent (ground) nodes represent decision inputs (from the sensors, satellites and other data sources) and other (the non-leaf and non-ground) nodes represent intermediate decision parameters. Some intermediate nodes that transform observed beliefs into actual beliefs are called the actual input (or actual belief) nodes.

Links between nodes identify causal-effect or dependency relationships that have been quantified by the use of conditional probability tables. The conditionals specify the prior joint distribution of the nodes. They also serve as means for coding explanation criteria. They enable a decision input (or state) to be preferred over another and also assign confidence values when combining them. They are also used to transform observed beliefs into actual beliefs. 
Figure 2 shows a section of the PRISM Bayesian network that determines the actual level of interest of an experiment in the site being measured. This influences the choice of the SAR mode (bistatic vs. monostatic). Four inputs, namely, the level of interest shown by the science community in the site $\left(X_{l}\right)$, PRISM SAR data $\left(X_{2}\right)$ and Satellite SAR data $\left(X_{3}\right)$ indicating the extent to which the site is a potential transition zone (a varying flow regime) or contains one or more transition zones, and the velocity magnitude or motion vector $\left(X_{4}\right)$ extracted from the Satellite SAR velocity maps are combined to yield an estimate of the experiment's actual level of interest (highly interesting, moderately interesting, not so interesting, etc.) in the site $\left(X_{5}\right)$.

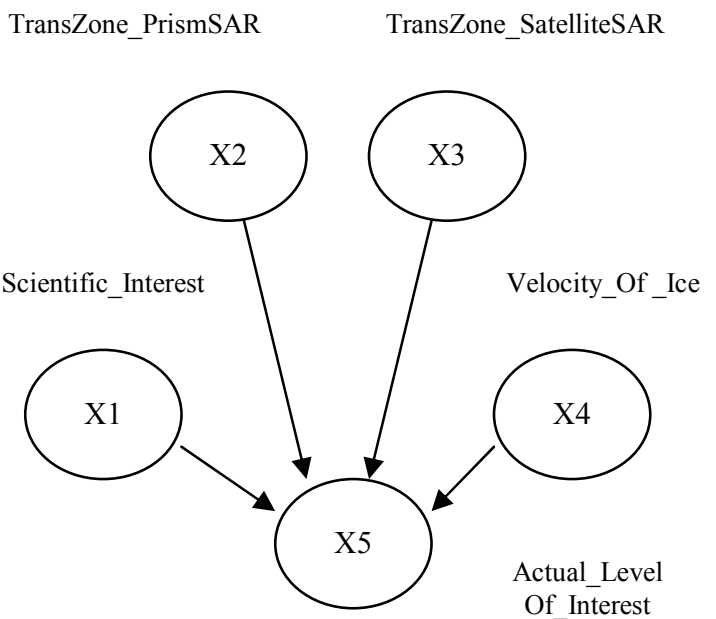

Figure 2. Example of a PRISM Bayesian network

When combining these decision input variables, $X_{I}$ is to be treated at a higher weight than $X_{2}$ and $X_{3}$ that are to be treated equal to each other and at a higher weight than $X_{4}$. Also, a value of " $\mathrm{H}$ " (high) for a decision input variable is to be weighed higher than " $M$ " (medium), which in turn is to be weighed higher than "L" (low). Such priority or preference over inputs and decision states is coded in terms of conditional probability values.

For example, if decision input variable $X_{I}$ is reported to be " $\mathrm{H}$ " then decision output variable $X_{5}$ is " $\mathrm{H}$ " irrespective of what is stated by the other input variables $X_{2}, X_{3}$ and $X_{4}$. But, if $X_{1}$ is reported to be "L", then the values of $X_{2}, X_{3}$ and $X_{4}$ will influence the value of $X_{5}$, with $X_{2}$ and $X_{3}$ influencing $X_{5}$ more than $X_{4}$. Thus,

$$
\begin{aligned}
& P\left(X_{5}=H \mid X_{1}=H, X_{2}=X, X_{3}=X, X_{4}=X\right)> \\
& P\left(X_{5}=H \mid X_{1}=L, X_{2}=H, X_{3}=H, X_{4}=X\right)> \\
& P\left(X_{5}=H \mid X_{1}=L, X_{2}=L, X_{3}=L, X_{4}=H\right)
\end{aligned}
$$

where a value of " $\mathrm{X}$ " for variables in equation (2) denotes "any" (H/M/L).

Table I and II show two different sections of the conditional probability table for the decision output variable $X_{5}$. Table I illustrates the case where variables $X_{2}, X_{3}$ and $X_{4}$ have very little influence on $X_{5}$, as $X_{1}$ is "high". Table II illustrates the scenario where variables $X_{2}, X_{3}$ and $X_{4}$ influence $X_{5}$, with $X_{1}$ being "low". Again, the influence of $X_{2}$ and $X_{3}$ on $X_{5}$ is greater than that of $X_{4}$ on $X_{5}$.

TABLE I. CONDITIONAL PROBABILITIES FOR NODE $\mathrm{X}_{5}$ WITH $\mathrm{X}_{1}=\mathrm{H}$

\begin{tabular}{|c|c|c|c|}
\hline X1 & \multicolumn{3}{|c|}{ High } \\
\hline X2 & \multicolumn{3}{|c|}{ Low } \\
\hline X3 & Low \\
\hline X4 & High & Medium & Low \\
\hline High & 0.92 & 0.90 & 0.88 \\
\hline Medium & 0.02 & 0.04 & 0.02 \\
\hline Low & 0.06 & 0.06 & 0.10 \\
\hline
\end{tabular}

TABLE II. CONDITIONAL PROBABILITIES FOR NODE $\mathrm{X}_{5}$ WITH $\mathrm{X}_{1}=\mathrm{L}$

\begin{tabular}{|c|c|c|c|}
\hline X1 & \multicolumn{3}{|c|}{ Low } \\
\hline X2 & \multicolumn{3}{|c|}{ High } \\
\hline X3 & High \\
\hline X4 & High & Medium & Low \\
\hline High & 0.70 & 0.65 & 0.65 \\
\hline Medium & 0.05 & 0.10 & 0.05 \\
\hline Low & 0.25 & 0.25 & 0.30 \\
\hline
\end{tabular}

Conditional probabilities enable transformation of observed beliefs into actual beliefs. For instance, the PRISM intelligent system associates a certain level of uncertainty to the measured (or observed) sensor and environmental input values, depending on the nature of the source/input. Such uncertainty can be coded and represented as conditionals in the conditional probability table of the corresponding ground node in the PRISM Bayesian network.

Table III shows one such conditional probability table. It is that of node "Actual Scientific Interest", and is based upon the prior probability distribution of the corresponding ground node "Observed Scientific Interest".

TABLE III. CONDITIONAL PROBABILITIES FOR AN ACTUAL INPUT NODE

\begin{tabular}{|l|c|c|c|}
\hline \multirow{2}{*}{ Actual } & \multicolumn{3}{|c|}{ Observed } \\
\cline { 2 - 4 } & High & Medium & Low \\
\hline High & 0.90 & 0.05 & 0.02 \\
\hline Medium & 0.08 & 0.90 & 0.08 \\
\hline Low & 0.02 & 0.05 & 0.90 \\
\hline
\end{tabular}

\section{IMPLEMENTATION \& WORKING}

The PRISM Bayesian network application is built upon the HUGIN Researcher V6.4 Java API library and is capable of inter-operating on the Linux and Windows platforms. The Bayesian network structure of nodes and edges has been constructed upon an object-oriented belief model comprising of HUGIN classes and class collections. Nodes and conditional probability tables have been defined using a special purpose 
language - called the NET language that is provided by the HUGIN system to textually specify a Bayesian network. The HUGIN API provides functions to parse and generate text files containing such descriptions. These files can also be interpreted by the HUGIN GUI, which can be used to view and edit the specified Bayesian network [6].

Intelligent agents receive and propagate input evidence (source data) in the Bayesian network as follows: They first analyze and transform source data into what are called as "decision states" or "input states". They then identify the corresponding input (parent) nodes in the Bayesian network and populate the nodes' conditional probability table by setting a value of 1 to the input state observed and a value of 0 to others. Observed inputs and beliefs are transformed into actual values based on the prior probabilities of the ground nodes and conditionals specified in the probability tables of the actual nodes. Finally, the intelligent agents call HUGIN functions to propagate evidence in the network and compute the posterior marginal probability distributions of the output nodes.

Evidence is propagated and the conditional probability distributions of the nodes are updated using the sequential learning method (also referred to as adaptation) developed by Spielgelhalter and Lauritzen [4][5] and implemented by the HUGIN system [6]. After propagation of evidence is complete, the revised beliefs or conditionals of the output nodes represent the certainty of the decision output, which is then treated as its rank (or risk value) that enables the choice of the best possible decision alternative.

\section{RESULTS \& CONCLUSION}

The complete PRISM intelligent system architecture and the Bayesian inference engine has been implemented and demonstrated on autonomous rovers and simulations of radar, and other data sources that have not been completed as of the writing of this paper. We have simulated the measurement and interpretation of such hypothetical and unavailable inputs using software "dummy" agents. For available inputs we have written "wrapper" agents to collect and distribute real-time data from existing sources.

Experiments have shown the feasibility of our approach and the success of our implementation, both in a general and in a near real-time setting. We deal well with most typical decisionmaking scenarios that may arise during the project's ice sheet measurements. The strength of probabilistic reasoning using Bayesian networks has effectively been demonstrated for the project. With source inputs being uncertain and changing unpredictably, the intelligent system comes up with the best possible decisions. Input evidence is being correctly entered and propagated. The conditional probability tables get effectively updated/adapted and yield correct and revised beliefs on propagation. The conditionals have effectively been used as means of specifying confidence in decision inputs/states and coding decision criteria and preference values.

The implementation and results prove significant especially in cases when the same property is being measured by more than one source and the values need to be combined to yield a composite estimate of the property. The system is to be extended to include real data sources that replace simulations of radar data and a few other rover sensors.

\section{ACKNOWLEDGEMENT}

We would like to acknowledge the efforts of John Paden, Hans Harmon, and the rest of the PRISM group for providing expert knowledge and support with the radar and rover inputs. We would also like to extend our heartfelt thanks to Steven Perry who has made a significant contribution to the project by expanding the multiagent collaborative architecture and developing intelligent agents for data collection, dissemination and delivery.

\section{REFERENCES}

[1] J. Paden. Bistatic/monostatic synthetic aperture radar for ice sheet measurements. Electrical engineering and computer science, University of Kansas, 1999.

[2] F. V. Jensen. An Introduction to Bayesian networks. Springer-Verlag, New York, 1999.

[3] F. V. Jensen. Bayesian Networks and Decision Graphs. Springer-Verlag, New York, 2001.

[4] R. G. Cowell, A. P. Dawid, S. L. Lauritzen, D. J. Spiegelhalter. Probabilistic Networks and Expert Systems. Statistics for Engineering and Information Science. Springer-Verlag, New York, 1999.

[5] D. J. Spiegelhalter and S. L. Lauritzen, "Sequential updating of conditional probabilities on directed graphical structures". Networks, 20(5), pp. 579-605, August 1990 [Special Issue on Influence Diagrams].

[6] Hugin Developer Tutorials and API Manuals. http://developer.hugin.com/. 